\title{
DIRETRIZES PARA PROGRAMAS DE GERENCIAMENTO DE RISCOS DE ACIDENTES AMBIENTAIS E OCUPACIONAIS (PGR) EM INSTALAÇÕES INDUSTRIAIS
}

\section{GUIDELINES FOR OF RISK MANAGEMENT PROGRAMS OF ENVIRONMENTAL AND OCCUPATIONAL ACCIDENTS (RMP) IN INDUSTRIAL PLANTS}

\author{
Eduardo Pavan Korf ${ }^{1}$; Claud Ivan Goellner ${ }^{2}$ \\ ${ }^{1}$ Universidade de Passo Fundo- UPF - Passo Fundo - RS - Brasil \\ eduardokorf@upf.br \\ ${ }^{2}$ Universidade de Passo Fundo- UPF - Passo Fundo - RS - Brasil \\ goellner@upf.br
}

\begin{abstract}
Resumo
O objetivo do trabalho foi apresentar diretrizes para elaboração de programas de gerenciamento de riscos de acidentes ambientais e ocupacionais (PGR) para aplicação em instalações industriais. As diretrizes apresentadas em um PGR abrangem o estudo de análise de risco, o plano de prevenção de risco, o plano de ação em emergências (PAE) e o plano de monitoramento ocupacional. O PGR constitui uma ferramenta indispensável para instalações que visem à implantação de sistemas de gestão integrada, bem como a certificação e o reconhecimento internacional. Além disso, a aplicação do PGR é vantajosa para aquelas instalações em que os riscos envolvidos são pouco conhecidos e as informações disponíveis são escassas.
\end{abstract}

Palavras-chave: ambiente; segurança humana; análise de riscos; prevenção de riscos; plano de ação de emergências.

\section{Introdução}

A importância dos acidentes ambientais e ocupacionais está relacionada com a evolução da atividade industrial e das relações de produção e consumo ao longo dos tempos. A evolução da natureza competitiva do setor industrial, aliada ao crescimento da economia mundial e ao avanço da tecnologia, possibilitou o aumento das plantas industriais e complexidade dos processos produtivos. O contexto social também foi se transformando e outros temas, tais como a poluição ambiental, segurança e saúde humana, começaram a se tornar motivo de preocupação para o público e para os governos. Como conseqüência, a indústria foi obrigada a examinar os efeitos de suas operações sobre o público e, em particular, a analisar mais cuidadosamente os possíveis perigos decorrentes de suas atividades (CETESB, 2009).

Segundo Dias (2001), os perigos, associados ao potencial de causar danos em instalações 
industriais e à saúde do trabalhador, podem ser caracterizados pela existência de agentes físicos, químicos, biológicos, ergonômicos ou psicossociais e mecânicos ou de acidentes. Todos esses agentes estão associados a um fator de risco, o qual expressa a possibilidade de ocorrência de um dado perigo. O risco de acidentes é, portanto, expressado em relação à freqüência ou probabilidade de ocorrência de um perigo e à magnitude (CAPONI, 2004).

Entre as décadas de 70 e 80 ocorreram uma série de acidentes que culminaram em grande impacto e reforçaram a necessidade de estudos na área de avaliação de riscos e prevenção de perdas, bem como a necessidade de estabelecimento de diretrizes, regulamentos e legislações sobre o tema, com o objetivo de reduzir ou evitar a ocorrência de acidentes industriais maiores (CETESB, 2009).

As principais ocorrências de acidentes ambientais tecnológicos no Brasil, segundo Ibama (2009) são derramamentos ou vazamentos de produtos nocivos, incêndios, explosões, descarrilamentos, colisões, etc. Dessa maneira, percebe-se que as principais causas de acidentes tecnológicos estão associadas às atividades industriais e atividades de transporte, também relacionadas aos processos industriais.

Segundo Gouveia e Nardocci (2007), os acidentes ambientais têm se caracterizado um passivo ambiental e de saúde pública devido à magnitude de suas conseqüências e complexidade de sua abordagem. Lainha (2003) aponta que essas ocorrências podem transformar-se em eventos agudos de poluição. Descargas acidentais e vazamentos podem gerar contaminações na atmosfera, com cargas tóxicas, inflamáveis e explosivas, bem como danos ao meio ambiente e à saúde dos trabalhadores e das comunidades expostas a seus efeitos.

O problema dos acidentes ambientais e ocupacionais pode ser analisado sob dois pontos: a sua magnitude e as características de sua ocorrência. O primeiro revela severas conseqüências ao meio ambiente e à segurança humana e o segundo apresenta como causa principal de ocorrência as atividades industriais.

Esse contexto justifica a necessidade de estudos investigativos envolvendo a análise dos riscos de acidentes e implantação de medidas de prevenção e gerenciamento dos riscos de ocorrência desses eventos. Segundo Caponi (2004), os estudos de análise de risco constituem uma ferramenta para elaboração de ações de prevenção de acidentes, que consiste em um estudo detalhado de um objeto, com a finalidade de identificar perigos e avaliar os riscos associados. Assim, o processo de avaliação de riscos utiliza os resultados da análise de riscos para a tomada de decisão quanto ao gerenciamento dos riscos, através da comparação com critérios de tolerabilidade de riscos previamente estabelecidos. Reis (2006) explica que os objetivos da execução de análise de riscos em instalações industriais podem ser: prevenir e prever falhas e acidentes, minimizar conseqüências e auxiliar na elaboração de planos de Ação de Emergência. Para isso, constitui-se 
necessária a adoção de metodologias sistemáticas e estruturadas para identificação de perigos, avaliação e gerenciamento de riscos.

Além disso, os resultados de um estudo de análise e avaliação de risco para prevenção e redução de acidentes constituem uma etapa de um programa de gerenciamento de riscos (PGR). Segundo Reis (2006), um programa de gerenciamento de riscos é uma ferramenta que deve ser implementada em atividades de instalações industriais que sejam vulneráveis a acidentes ao longo de sua vida útil. Maschio (2007) apresenta que as etapas de um PGR podem ser divididas em: identificação e avaliação de riscos, quantificação, monitoramento e controle. O PGR, de acordo com Reis (2006), tem o objetivo de desenvolver uma sistemática voltada para o estabelecimento de requisitos, que sirvam como orientações gerais de gestão, no sentido da prevenção de acidentes, bem como atendimento de emergências.

O objetivo do trabalho foi apresentar diretrizes para elaboração de programas de gerenciamento de riscos de acidentes ambientais e ocupacionais (PGR) para aplicação em instalações industriais.

\section{Metodologia}

As diretrizes e a proposta de estrutura para a elaboração de um programa de gerenciamento de riscos (PGR) foram elaboradas seguindo elementos apresentados por Fepam (2001) e Reis (2006). A proposta de técnica de análise de risco, a qual é parte integrante do PGR, foi baseada nos dois primeiros níveis de análise de risco abordada por Environmental Agency (2004) e adaptada de técnica de análise preliminar de perigo (APP) e análise preliminar de perigo e pontos críticos de controle (APPCC), as quais são apresentadas na literatura por diversos autores (AGUIAR, 2009; MINISTÉRIO DE AGRICULTURA E DO ABASTECIMENTO, 2009; FAXINA, 2008; SILBERMAN; MATTOS, 2008; REIS, 2006; SENAC/DN, 2002; FEPAM, 2001; BRASIL, 1998).

Este metodologia apresentada se enquadra na situação de estudo, pois a área de abrangência de um PGR busca nortear tanto a construção de um plano de ações preventivas quando ações de atendimento emergencial. Dentro da abrangência de um PGR, estudos de análise de risco são para instalações que almejam uma análise de caráter inicial. A aplicação da técnica de APPCC para levantamento de perigos e riscos é uma revisão de problemas gerais de segurança de uma instalação, que constitui uma análise inicial que possui especial importância na investigação de sistemas pouco conhecidos, ou seja, quando a experiência em riscos na sua operação é carente ou deficiente. Esta técnica pode preceder uma avaliação puramente quantitativa de risco subseqüente, quando esta se fizer necessária (REIS, 2006).

\section{Resultados e discussão - diretrizes para elaborar de um PGR}


Na sequência serão detalhadas cada um das etapas que devem compor um PGR.

\subsection{Apresentação}

Nesta etapa deve ser apresentada a essência de um PGR, justificada a importância de sua implantação em instalações industriais e detalhadas as finalidades como os objetivos e metas a serem alcançadas.

\subsection{Alocação de responsabilidades e atribuições}

Para implantação do PGR, segundo Reis (2006), é necessário designar responsabilidades e atribuições a uma equipe. A estrutura organizacional do PGR deverá ser montada e devem ser relacionadas todas as equipes e enquadramentos funcionais responsáveis pela implementação de cada um dos elementos do PGR (FEPAM, 2001; REIS, 2007).

Um fluxograma que apresente a estrutura organizacional do PGR é fundamental para mostrar o ordenamento das equipes responsáveis pela implantação e execução do PGR. Recomenda-se nesta etapa a elaboração de um quadro que relacione a equipe, o enquadramento funcional e os componentes responsáveis nos diferentes turnos de funcionamento da empresa. Além disso, deverão ser designadas e detalhadas as atribuições para cada um dos enquadramentos funcionais da estrutura organizacional.

\subsection{Caracterização da Instalação}

Nesta etapa deverá ser caracterizada a instalação, detalhando as seguintes informações:

a) Localização da Instalação;

b) Visão de mercado e área de atuação da Empresa;

c) Detalhamento de todas as linhas dos processos produtivos, apresentando as operações unitárias envolvidas e os procedimentos operacionais;

d) Detalhamento dos setores de apoio ao processo produtivo da indústria;

e) Apresentação de fluxogramas de linhas do processo produtivo e setores da empresa;

f) Apresentação de vista aérea do empreendimento e de planta baixa apresentando a localização das linhas e setores de apoio;

g) Apresentação de diagnóstico ambiental da empresa, detalhando: os setores de meio ambiente e segurança do trabalho na estrutura organizacional da empresa; as ações de segurança, meio ambiente e controle ambiental da poluição no processo produtivo e setores de apoio;

h) Caracterização da região de entorno à instalação em um raio mínimo de $1000 \mathrm{~m}$, acompanhada de cartas, mapas, plantas e fotografias aéreas as quais indiquem: representação do entorno no meio urbano, detalhando o zoneamento municipal em termos de regiões residenciais 
existentes, presença de outras indústrias, áreas rurais, etc. e detalhamento da área da indústria no zoneamento do plano diretor municipal; em caso de zona residencial quantificar a ocorrência unidades residenciais, de comércio, prestação de serviço, entre outras; detalhamento do meio físico indicando presença de corpos hídricos superficiais e sua localização em bacias hidrográficas específicas, poços de abastecimento de água subterrânea, áreas de preservação, presença de nascentes e banhados, unidades de conservação, uso do solo, relevo existente e características do solo.

\subsection{Análise de riscos de acidentes}

A proposta a ser desenvolvida nesta etapa é composta por dois níveis de análise, conforme divisão abordada por Environmental Agency (2004) e adaptação de técnica apresentada na literatura por diversos autores (Aguiar, 2009; Brasil, 1998; Faxina, 2008; Fepam, 2001; Ministério de Agricultura e do Abastecimento, 2009; Reis, 2006; Senac/Dn, 2002; Silberman e Mattos, 2008). Nesta etapa também deve ser apresentada a área de abrangência da análise de risco na instalação industrial.

A Figura 1 apresenta as etapas da análise de risco para a metodologia proposta.

Figura 1 - Etapas de análise de risco

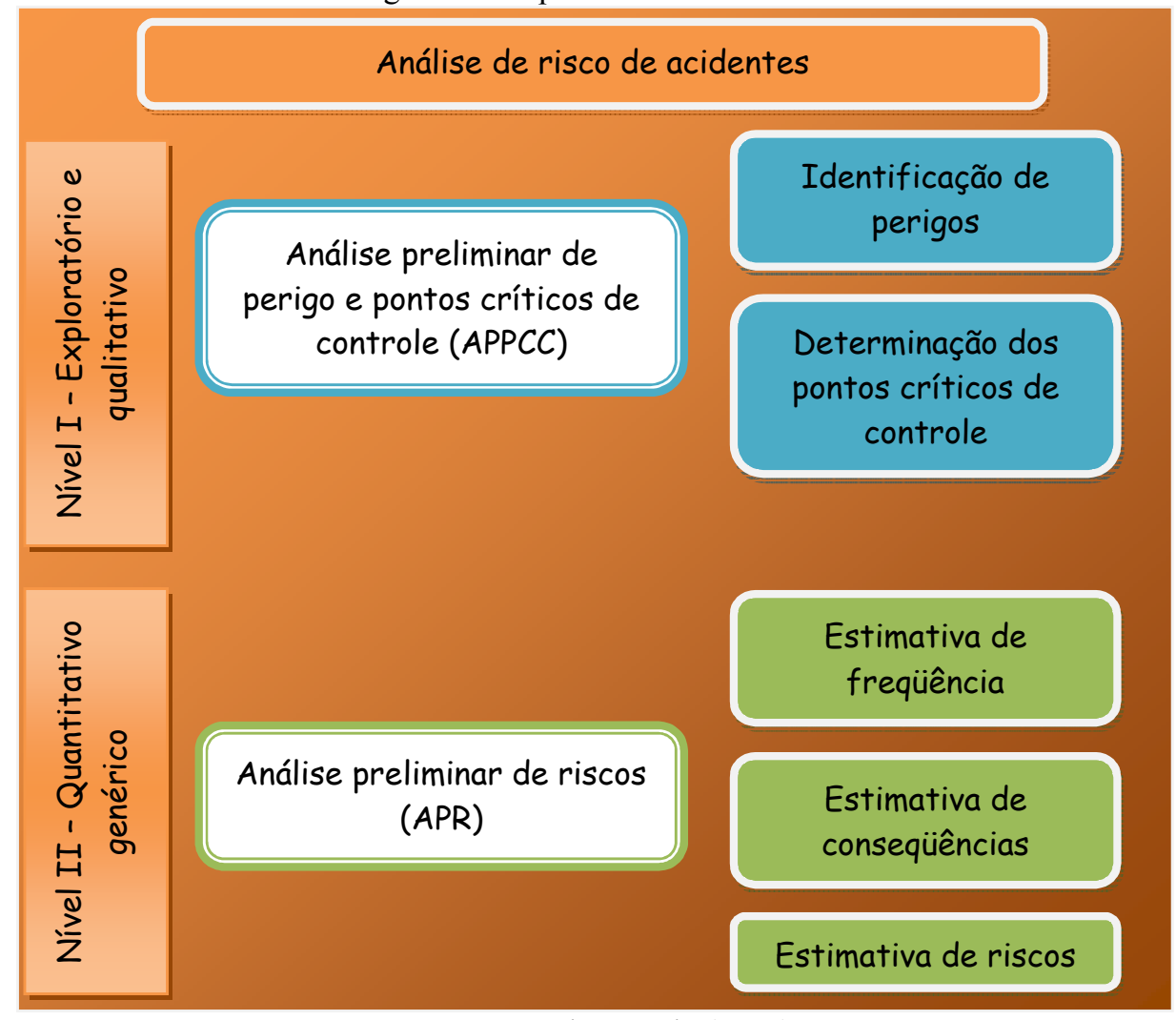

Fonte: Autoria própria (2009)

\subsubsection{Nível I - Avaliação exploratória e qualitativa}


Este nível compõe uma técnica de caráter exploratório e qualitativo, a qual constitui uma análise preliminar de perigo e pontos críticos de controle (APPCC). Deve ser aplicado um inventário na planta industrial, identificando em cada linha do processo produtivo ou setor, perigos envolvidos e para cada um deles, pontos críticos de controle.

O inventário de perigos e pontos críticos de controle pode ser realizado através de realização de diversas visitas de cunho técnico em cada processo e/ou setor da empresa. Para cada operação unitária das linhas do processo produtivo e para cada setor de apoio devem ser identificados perigos inerentes à instalação que podem ser causados por agentes de natureza física, química, biológica, ergonômica, mecânica e de acidentes. A partir do inventário de perigos, devem ser relacionados pontos críticos de controle (PCC), que são pontos de cada processo ou setor em que devem ser estabelecidas ações de controle. Maiores informações sobre a descrição dos agentes causadores de perigos podem ser encontradas em (DIAS, 2001; FILHO, 1999; HIRATA, 2002; NETO, 2006). O Quadro 1 apresenta a descrição dos agentes causadores de perigos em instalações apresentados por (DIAS, 2001; FILHO, 1999; HIRATA, 2002; NETO, 2006). O Quadro 2 apresenta exemplos de perigos identificados e pontos críticos de controle (PCC) relacionados.

Quadro 1 - Descrição dos agentes causadores de perigos em instalações

\begin{tabular}{|c|c|}
\hline Agente & Descrição \\
\hline \multirow{8}{*}{ Físico } & Calor \\
\hline & Frio \\
\hline & Radiações Ionizantes \\
\hline & Radiações não-ionizantes \\
\hline & Vibrações \\
\hline & Umidade \\
\hline & Pressão anormais \\
\hline & Ruído \\
\hline \multirow{12}{*}{ Químico } & Poeiras \\
\hline & Fumaças \\
\hline & Fumos Metálicos \\
\hline & Névoa \\
\hline & Neblinas \\
\hline & Vapores Orgânicos \\
\hline & Gases inertes \\
\hline & Gases/vapores ácidos \\
\hline & Gases/vapores alcalinos \\
\hline & Gases/vapores organometálicos \\
\hline & Gases/vapores especiais \\
\hline & Manipulação de substâncias químicas \\
\hline \multirow{4}{*}{ Biológicos } & Vírus \\
\hline & Fungos \\
\hline & Protozoários \\
\hline & Bactérias \\
\hline
\end{tabular}


Quadro 1 - Descrição dos agentes causadores de perigos em instalações (continuação)

\begin{tabular}{|c|c|}
\hline Agente & Descrição \\
\hline \multirow{4}{*}{ Ergonômicos e Psicossociais } & Trabalhos físicos pesados \\
\cline { 2 - 2 } & Posturas inadequadas e incômodas \\
\cline { 2 - 2 } & Ritmos excessivos \\
\cline { 2 - 2 } & Monotonia \\
\cline { 2 - 2 } & Trabalho em turnos \\
\cline { 2 - 2 } & Jornada prolongada \\
\cline { 2 - 2 } & Conflitos \\
\cline { 2 - 2 } & Remuneração por produtividade \\
\cline { 2 - 2 } & Arranjo físico inadequado \\
\cline { 2 - 2 } & Máquinas sem proteção \\
\cline { 2 - 2 } & Instalações elétricas inadequadas e desprotegidas \\
\cline { 2 - 2 } & Ferramentas inadequadas \\
\cline { 2 - 2 } & Piso/degrau escorregadio ou irregular \\
\cline { 2 - 2 } & Equipamentos e instrumentos pérfurocortantes \\
\cline { 2 - 2 } & \\
\cline { 2 - 2 } &
\end{tabular}

Fonte: Autoria própria (2009)

Quadro 2 - Exemplos de perigos identificados e PCCs relacionados

\begin{tabular}{|c|c|}
\hline Perigo & PCC \\
\hline \multirow{3}{*}{$\begin{array}{l}\text { Transbordo de efluente no bombeamento da } \\
\text { Estação de tratamento de efluentes, podendo } \\
\text { ocorrer vazamento para o ambiente }\end{array}$} & Falha na bóia de acionamento da bomba de saída \\
\hline & $\begin{array}{l}\text { Falha no funcionamento do alarme de alerta de nível crítico do } \\
\text { efluente da caixa de saída }\end{array}$ \\
\hline & Falha no bombeamento de efluente \\
\hline \multirow{4}{*}{$\begin{array}{l}\text { Exposição de soda cáustica ao organismo } \\
\text { humano e derramamento no ambiente }\end{array}$} & Manuseio devido à realização de análises químicas \\
\hline & Manuseio no pátio da estação de tratamento \\
\hline & Limpeza dos tachos de cozimento \\
\hline & $\begin{array}{l}\text { Acondicionamento inadequado na central de tratamento de resíduos } \\
\text { sólidos }\end{array}$ \\
\hline
\end{tabular}

Fonte: Autoria própria (2009)

\subsubsection{Nível II - Avaliação quantitativa genérica}

Nesta etapa, para cada perigo e ponto crítico identificado, deve-se aplicar a metodologia quali-quatitativa ou quantitativa genérica de análise preliminar de riscos (APR). Para a análise deve ser realizada a estimativa da freqüência e a estimativa de conseqüências, para, por fim, se realizar a estimativa de riscos.

\subsubsection{Estimativa da frequência}

A estimativa quantitativa da freqüência para cada ponto crítico deve ser realizada de acordo com as categorias de freqüência sugeridas por Fepam (2001) e por faixas de valores apresentados por Aguiar (2009). Nesta faixa de valores estabelecida, houve uma adaptação de Aguiar (2009) sendo que foi adotado o extremo superior da faixa estabelecida. O Quadro 3 apresenta as categorias de freqüência sugeridas e valores para serem adotados para estimativa. 
Quadro 3 - Categorias de freqüência utilizadas e valores adotados

\begin{tabular}{|c|c|c|c|}
\hline Categoria & Denominação & \multicolumn{1}{c|}{ Descrição } & $\begin{array}{c}\text { Valores } \\
\text { (ano-1 }^{-1}\end{array}$ \\
\hline A & Muito Improvável & $\begin{array}{l}\bullet \text { Falhas múltiplas de sistemas de proteção ou ruptura por falha } \\
\text { mecânica de vasos de pressão; } \\
\text { Conceitualmente possível, mas extremamente improvável de ocorrer } \\
\text { durante a vida útil da instalação. }\end{array}$ & $1.10^{-4}$ \\
\hline B & Improvável & $\begin{array}{l}\text { - Falhas múltiplas no sistema (humanas e/ou equipamentos) ou } \\
\text { rupturas de grande porte; } \\
\text { - Não esperado ocorrer durante a vida útil da instalação. Sem registro } \\
\text { de ocorrência prévia na instalação. }\end{array}$ & $1.10^{-3}$ \\
\hline C & Ocasional & $\begin{array}{l}\bullet \text { A ocorrência do cenário depende de uma única falha (humana ou } \\
\text { equipamento). }\end{array}$ & $1.10^{-2}$ \\
\hline D & Provável & $\bullet$ Esperada uma ocorrência na vida útil do sistema. & $1.10^{-1}$ \\
\hline E & Freqüente & $\begin{array}{l}\text { - Pelo menos uma ocorrência já registrada; } \\
\bullet \text { Esperado ocorrer várias vezes na vida útil do sistema. }\end{array}$ & 1 \\
\hline
\end{tabular}

Fonte: Baseada em Informações de Fepam (2001) e Aguiar (2009)

\subsubsection{Estimativa das conseqüências}

A análise qualitativa das conseqüências de cada acidente ocasionado por cada ponto crítico de controle deve ser realizada através de estudo das conseqüências apontadas na literatura. No caso de acidentes causados por agentes físicos, mecânicos e de acidentes, ergonômicos e biológicos, as possíveis conseqüências podem ser levantadas a partir de informações trazidas por (DIAS, 2001; FILHO, 1999; HIRATA, 2002; NETO, 2006). No caso de acidentes causados por agentes químicos de característica perigosa, as possíveis conseqüências podem ser levantadas a partir de informações apresentadas por Canutec Ergo (2008), software Emergency Response Guidebook e em fichas de informação de segurança de produtos químicos (FISPQ), os quais relacionam as informações toxicológicas, ecotoxicológicas e ambientais para cada tipo de produto perigoso. No caso de acidentes causados por agentes químicos de característica não perigosa, as possíveis conseqüências podem ser levantadas a partir das fichas de informação de segurança de produtos químicos (FISPQ), fornecidas pelos fabricantes dos produtos, a qual relaciona as informações toxicológicas, ecotoxicológicas e ambientais de cada tipo de produto químico.

A estimativa quantitativa de conseqüência e vulnerabilidade de cada ponto crítico de controle (PCC) deve ser realizada de acordo com as categorias de indicação de severidade de conseqüências apresentadas por Fepam (2001). Como proposta de aplicação, foram estabelecidos valores para cada categoria de modo a atingir o alcance de valores de risco aceitáveis e não aceitáveis, para cada classe de risco que será definida posteriormente. O Quadro 4 apresenta as categorias de severidade utilizadas para avaliação das conseqüências e os valores adotados para estimativa. 
Quadro 4 - Categorias de severidade para avaliação de conseqüência e valores adotados

\begin{tabular}{|c|c|l|c|}
\hline Categoria & Denominação & \multicolumn{1}{|c|}{ Descrição } & Valores* \\
\hline I & Desprezível & $\begin{array}{l}\text { - Incidentes com causa de indisposição ou mal-estar ao pessoal e } \\
\text { danos insignificantes ao ambiente; } \\
\text { - Sem impactos ambientais. }\end{array}$ & $10^{-2}$ \\
\hline II & Marginal & $\begin{array}{l}\text { - Potencial de causar ferimentos ao pessoal, pequenos danos ao } \\
\text { ambiente ou equipamentos/instrumentos, redução significativa da } \\
\text { produção; } \\
\text { - Impactos ambientais restritos ao local, controlável. }\end{array}$ & $10^{-1}$ \\
\hline III & Crítica & $\begin{array}{l}\text { - Potencial de causar uma ou algumas vítimas fatais ou grandes } \\
\text { danos ao ambiente e instalações; } \\
\text { - Ações corretivas imediatas para evitar catástrofe. }\end{array}$ & 1 \\
\hline IV & Catástrófica & $\begin{array}{l}\text { - Potencial para causar várias vítimas fatais; } \\
\text { - Danos irreparáveis ou impossíveis (custo/tempo) às instalações. }\end{array}$ & 10 \\
\hline
\end{tabular}

Fonte: Baseada em informações da Fepam (2001). *Adimensional

\subsubsection{Estimativa de riscos}

A definição de risco é a combinação entre a freqüência $(f)$ de um evento pela conseqüência de seus impactos gerados $(C)$, conforme apresentado pela equação 1, segundo (SILBERMAN; MATTOS, 2008).

$$
\text { RISCO }=f \times C
$$

A estimativa de risco deve ser realizada através da combinação entre as categorias de risco sugeridas pela Fepam (2001), gerando uma matriz de classificação de risco. A Fepam (2001) apresenta 5 classes resultantes da combinação entre as 5 categorias de freqüência e as 4 categorias de conseqüência. Na matriz, os valores propostos, utilizados para o cálculo do risco em cada classe de risco, obedeceram ao critério de aceitabilidade de risco individual adotado pela Inglaterra, apresentado por Silberman e Mattos (2008), o qual considera o nível máximo tolerável (ocupacional) para trabalhadores de $1.10^{-3}$ ano, o que significa 1 ocorrência de um dado evento em 1000 anos. Das 5 classes de risco adotadas pela Fepam (2001), considerou as três últimas como categorias não aceitáveis, ou seja, que possuem valores acima do nível máximo estabelecido. O Quadro 5 apresenta a matriz de classificação apresentada pela Fepam (2001) adaptada com os valores estabelecidos para quantificação. 
Quadro 5 - Matriz de classificação de risco com valores estabelecidos para quantificação

\begin{tabular}{|c|c|c|c|c|c|c|c|c|}
\hline & & & \multicolumn{6}{|c|}{ Categoria de Conseqüência (valores) } \\
\hline & & & & I $\left(1.10^{-2}\right)$ & II $\left(1.10^{-1}\right)$ & & & IV (10) \\
\hline \multirow{5}{*}{\multicolumn{2}{|c|}{ 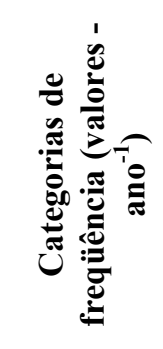 }} & E (1) & & $3\left(1.10^{-2}\right)$ & $4\left(1.10^{-1}\right)$ & & & $5(10)$ \\
\hline & & $D\left(1.10^{-1}\right)$ & & $2\left(1.10^{-3}\right)$ & $3\left(1.10^{-2}\right)$ & & $\left.0^{-1}\right)$ & $5(1)$ \\
\hline & & $C\left(1.10^{-2}\right)$ & & $1\left(1.10^{-4}\right)$ & $2\left(1.10^{-3}\right)$ & & $\left.0^{-2}\right)$ & $4\left(1.10^{-1}\right)$ \\
\hline & & B $\left(1.10^{-3}\right)$ & & $1\left(1.10^{-5}\right)$ & $1\left(1.10^{-4}\right)$ & & $\left.0^{-3}\right)$ & $3\left(1.10^{-2}\right)$ \\
\hline & & $A\left(1.10^{-4}\right)$ & & $1\left(1.10^{-6}\right)$ & $1\left(1.10^{-5}\right)$ & & $\left.0^{-4}\right)$ & $2\left(1.10^{-3}\right)$ \\
\hline \multicolumn{3}{|c|}{ Freqüência } & \multicolumn{3}{|c|}{ Conseqüência } & \multicolumn{3}{|c|}{ Risco } \\
\hline A & Muito & $\begin{array}{l}\text { vável }\left(1.10^{-4}\right. \\
\left.\mathrm{o}^{-1}\right)\end{array}$ & I & \multicolumn{2}{|c|}{ Desprezível $\left(1.10^{-2}\right)$} & 1 & \multicolumn{2}{|c|}{ Desprezível $\left(<10^{-3}\right.$ ano $\left.^{-1}\right)$} \\
\hline B & & $\begin{array}{l}\text { ovável } \\
\left.\text { ano }^{-1}\right)\end{array}$ & II & \multicolumn{2}{|c|}{ Marginal $\left(1.10^{-1}\right)$} & 2 & \multicolumn{2}{|c|}{ Menor $\left(10^{-3} \mathrm{ano}^{-1}\right)$} \\
\hline $\mathrm{C}$ & & $\begin{array}{l}\text { ional } \\
\left.\text { ano }^{-1}\right)\end{array}$ & III & \multicolumn{2}{|c|}{ Crítica (1) } & 3 & \multicolumn{2}{|c|}{ Moderado $\left(10^{-2}\right.$ ano $\left.^{-1}\right)$} \\
\hline $\mathrm{D}$ & & $\begin{array}{l}\text { vável } \\
1 \text { ano }^{-1} \text { ) } \\
\end{array}$ & IV & \multicolumn{2}{|c|}{ Catastrófica (10) } & 4 & \multicolumn{2}{|c|}{ Sério $\left(10^{-1}\right.$ ano $\left.^{-1}\right)$} \\
\hline $\mathrm{E}$ & \multicolumn{2}{|c|}{ Freqüente $\left(1\right.$ ano $\left.^{-1}\right)$} & & & & 5 & \multicolumn{2}{|c|}{ Crítico $\left(>10^{-1}\right.$ ano $\left.^{-1}\right)$} \\
\hline \multicolumn{3}{|c|}{ Tipo de risco } & \multicolumn{3}{|c|}{ Categoria } & \multicolumn{3}{|c|}{ Valor } \\
\hline \multirow{2}{*}{\multicolumn{3}{|c|}{ Aceitável }} & \multicolumn{3}{|c|}{1 - Desprezível $\left(<10^{-4}\right.$ ano $\left.^{-1}\right)$} & \multirow{2}{*}{\multicolumn{3}{|c|}{ Risco $\leq 10^{-3}$ ano $^{-1}$}} \\
\hline & & & \multicolumn{3}{|c|}{$2-$ Menor $\left(10^{-3}\right.$ ano $\left.^{-1}\right)$} & & & \\
\hline \multirow{3}{*}{\multicolumn{3}{|c|}{ Não Aceitável }} & \multicolumn{3}{|c|}{3 - Moderado $\left(10^{-2}\right.$ ano $\left.^{-1}\right)$} & \multirow{3}{*}{\multicolumn{3}{|c|}{ Risco $>10^{-3}$ ano $^{-1}$}} \\
\hline & & & \multicolumn{3}{|c|}{4 - Sério $\left(10^{-1}\right.$ ano $\left.^{-1}\right)$} & & & \\
\hline & & & \multicolumn{3}{|c|}{5 - Crítico $\left(>1\right.$ ano $\left.^{-1}\right)$} & & & \\
\hline
\end{tabular}

Fonte: Adaptada de FEPAM (2001)

O Quadro 6 apresenta modelo de planilha que pode ser utilizada para inventário de perigos nos setores e identificação dos riscos associados após análise de risco. O Quadro 7 apresenta modelo de planilha que pode ser utilizada para a aplicação da metodologia de análise preliminar de risco.

Quadro 6 - Planilha para inventário de perigos e identificação de riscos após análise

\begin{tabular}{|c|c|c|c|c|c|c|c|c|}
\hline \multicolumn{3}{|c|}{ Risco Associado } & \multirow{3}{*}{ 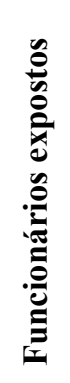 } & $\mathbf{A}$ & $\mathbf{A}$ & B & D & $\mathbf{A}$ \\
\hline \multicolumn{3}{|c|}{ Agente } & & Físico & Químico & Mecânico & Físico & Biológico \\
\hline & & & & & & & & \\
\hline & & & & & & & & \\
\hline
\end{tabular}

Fonte: Autoria própria (2009) 
Quadro 7 - Planilha para aplicação de metodologia de análise de risco

\begin{tabular}{|l|l|c|c|c|c|c|c|}
\hline Perigo & PCC & $\begin{array}{c}\text { Agente } \\
\text { causador }\end{array}$ & $\begin{array}{c}\text { Conseqüência de } \\
\text { acidentes }\end{array}$ & $\begin{array}{c}\text { Freqüência } \\
\left(\text { ano }^{-1}\right)\end{array}$ & Conseqüência & $\begin{array}{c}\text { Quantificação do } \\
\text { risco } \\
\left(\text { ano }^{-1}\right)\end{array}$ & $\begin{array}{c}\text { Categoria } \\
\text { de risco }\end{array}$ \\
\hline & & & & & & & \\
\hline
\end{tabular}

Fonte: Autoria própria (2009)

\subsection{Gerenciamento de riscos de acidentes}

A partir da análise de risco realizada, o gerenciamento dos riscos de acidentes deverá ser realizado por priorização, desde a categoria de risco “crítica” até a categoria "desprezível”. Deve ser elaborado um quadro estabelecendo a ordem de prioridade para controle de ações em cada PCC e a indicação dos tipos de ações de controle que devem ser elaboradas, designadas como ações preventivas e ações emergenciais. O Quadro 8 apresenta um modelo de planilha de gerenciamento de riscos de acidentes e priorização das ações. A ordem de prioridade que deve ser utilizada deve ser correlacionada com as categorias de risco.

Quadro 8 - Planilha para gerenciamento de riscos de acidentes e priorização de ações

\begin{tabular}{|c|c|c|c|c|}
\hline Perigo & PCC & Ordem de prioridade & Ações preventivas & Ações emergenciais \\
\hline & & & & \\
\hline
\end{tabular}

Fonte: Autoria própria (2009)

\subsection{Plano de prevenção de riscos de acidentes}

O plano de prevenção de riscos de acidentes deve considerar o detalhamento de todas as necessidades de ações preventivas apontadas para cada perigo e PCC, na planilha de gerenciamento de riscos. A estrutura do plano deverá apresentar:

a) Cenários considerados, área de abrangência e limitações;

b) Recursos humanos e materiais necessários;

c) Medidas de controle, redução e prevenção de riscos.

\subsection{Plano de ação de emergência (PAE)}

O plano de ação de emergência deve considerar o detalhamento de todas as necessidades de ações emergenciais apontadas para cada perigo e PCC, na planilha de gerenciamento de riscos. A estrutura do plano deverá apresentar:

a) Cenários considerados, área de abrangência e limitações;

b) Centro de controle de emergência e estrutura organizacional;

c) Recursos humanos e materiais;

d) Fluxograma de acionamento;

e) Ações de resposta;

f) Treinamentos e simulações; 

g) Comunicação;
h) Investigação de acidentes.

\subsection{Plano de monitoramento da saúde ocupacional}

O plano de monitoramento da saúde ocupacional deve considerar todo o monitoramento de todos e qualquer agente de perigo capazes de causar aos distúrbios à saúde do trabalhador devido à sua exposição com característica crônica, ou seja, a qual se caracteriza pela exposição à baixas doses e por evidência de sintomas em longo prazo. A estrutura do plano deverá apresentar:

a) Cenários considerados;

b) Ações de monitoramento.

\subsection{Registro, avaliação, manutenção e comunicação}

Neste item devem ser apresentadas formas de registro para todas as ações desenvolvidas dentro da empresa em relação ao PGR. Também deverão ser estabelecidos procedimentos de registro de todos os acidentes ocorridos e este registro deve ser conduzido à avaliação.

Os procedimentos de avaliação deverão representar ações que serão executadas durante o registro e avaliação de acidentes e por ações revisão ou manutenção que devem ser executadas periodicamente de modo a realizar melhorias, alterações e correções ao PGR.

Os documentos gerados e toda a composição do PGR enquanto plano e nas atividades de implantação deverão possuir controle interno e externo de modo a preservar documentos originais, cópias controladas e não controladas e controlar o número de cópias existentes.

Os procedimentos de comunicação deverão apresentar e definir forma de abordagem de informações relativas a PGR tais como: ações preventivas e emergenciais realizadas, treinamentos e simulações, etc. Além disso, a comunicação deverá servir como ferramenta de implantação do PGR, explicando sobre o seu funcionamento. As formas de comunicação poderão ter abrangência interna e externa.

\section{Conclusões}

O Programa de Gerenciamento de Riscos (PGR) constitui uma ferramenta indispensável e de fundamental importância para instalações que visem à implantação de sistemas de gestão integrada, tais como sistema de gestão ambiental e de segurança ocupacional, bem como certificação e reconhecimento internacional. A metodologia desenvolvida é vantajosa para instalações em que os conhecimentos dos riscos envolvidos são pouco conhecidos e as informações disponíveis são escassas. Com a consolidação do PGR, as instalações industriais poderão exercer 
ações preventivas, bem como exercer o adequado atendimento a emergências de acidentes contribuindo, assim, para a segurança ocupacional e ambiental.

\section{Abstract}

The goal was to provide guidelines for development of risk management programs of environmental and occupational accidents (RMP) for use in industrial plants. The guidelines presented in a RMP include the study of risk analysis, the plan for risk prevention, the plan for emergency response (PER) and occupational monitoring plan. RMP is an essential tool for industrial plants that require implementation of total environmental quality programs, as well as certification and international recognition. Furthermore, this methodology is advantageous for those installations where the risk are fewer known and available data are poor.

Key-words: environment; human security; risk analysis; risk prevention; plan for emergency response (per).

\section{Referências}

AGUIAR, L. A. de. Metodologias de análise de risco: APP \& HAZOP. Disponível em:

$<$ http://www.saneamento.poli.ufrj.br/documentos/Josimar/APP_e_HAZOP.pdf>. Acesso em: 13 set. 2009.

ALBERTON, A. Uma metodologia para auxiliar no gerenciamento de riscos e na seleção de alternativas de investimentos em segurança. Dissertação (Mestrado) -Engenharia de Produção, Universidade Federal de Santa Catarina, Florianópolis - SC., 1996.

BRASIL, MINISTÉRIO DA AGRICULTURA, PECÚARIA E ABASTECIMENTO. Portaria nº 46 de 10 de fevereiro de 1998. Institui o sistema de análise de perigo e pontos críticos de controle - APPCC a ser implantado gradativamente, nas indústrias de produto de origem animal sob o regime de serviço de inspeção federal - SIF, de acordo com o manual genérico de procedimentos. Diário oficial da República Federativa do Brasil. Brasília, DF, 16. Mar. 1998.

CANUTEC ERGO. (2008). ERGO 2008.exe: Emergency response Guidebook. Version 1.2.0.0. Ottawa - Canadá. Transport Canadá. Disponível em: <http://www.tc.gc.ca/canutec/en/GUIDE/ergo.htm>. Acesso em: 8 Jul. 2009.

CAPONI, A. C. Proposta de método para identificação de perigos e para avaliação e controle de riscos na construção de edificações. Dissertação (mestrado) - Universidade Estadual de Campinas, Faculdade de Engenharia Civil, Arquitetura e Urbanismo. Campinas - SP: UNICAMP, 173 p., 2004.

CETESB. Atendimento a acidentes com produtos químicos. São Paulo. CETESB, 59 p., 1993.

Estudos de análise de riscos: Histórico. Disponível em: $<$

http://www.cetesb.sp.gov.br/emergencia/riscos/estudo/historico.asp >. Acesso em: 8 Set. 2009.

DIAS, E. C. D (Org.). Doenças relacionadas ao trabalho: manual de procedimentos para os serviços de saúde. Brasília - DF. Ministério da Saúde do Brasil, 580 p., 2001.

ENVIRONMENTAL AGENCY. (2004). Guidance on the assessment of risks from landfill sites. Disponível em: $<$ http://www.environment-agency.gov.uk/static/documents/Research/risk_a_landfills_v1_768278.pdf >. Acesso em: 19 set. 2009.

FAXINA, F. L. Elaboração do plano de Análise de Perigos e Pontos Críticos de Controle (APPCC) para produção de Aveia em flocos na empresa Nat Cereais e Alimentos Ltda. Relatório de estágio curricular (Curso de Engenharia de Alimentos) - Faculdade de Engenharia e Arquitetura, Universidade de Passo Fundo, Passo Fundo - RS, 47 p., 2008.

FEPAM - FUNDAÇÃO ESTADUAL DE PROTEÇÃO AMBIENTAL HENRIQUE ROESSLER. Manual de análise de riscos. Rio Grande do Sul, 45 p, 2001.

FILHO, A. L. da S. Segurança química: risco químico no meio ambiente de trabalho. São Paulo. LTr, 136 p., 1999. 
GOUVEIA, J. L. N.; NARDOCCI, A. C. Acidentes em postos e sistemas trabalhistas de combustíveis: subsídios para vigilância em saúde ambiental. Revista de Engenharia Sanitária e Ambiental, v.12, n.3, p. 317-324, 2007.

HIRATA, M. H. O laboratório de ensino e pesquisa e seus riscos. In: HIRATA, M. H.; Manual de biossegurança. Barueri - SP. Manole, 496 p., 2002.

INSTITUTO BRASILEIRO DE MEIO AMBIENTE. Emergências e acidentes ambientais. Disponível em: $<$ http://www.ibama.gov.br/cgzam/>. Acesso em: 5 set. 2009.

LAINHA, M. A. J. Sistema integrado de gestão para prevenção, preparação e resposta aos acidentes com produtos químicos: manual de orientação. São Paulo.

CETESB - OPAS/OMS, 45 p., 2003.

MASCHIO, A. Gerenciamento de risco e segurança: Aplicabilidade e importância para o sucesso de projetos. Dissertação (mestrado) - Universidade Federal do Rio Grande do Sul, Escola de Engenharia, Programa de pósgraduação em Engenharia de produção. Porto Alegre: UFRGS, 141 p., 2007.

MINISTÉRIO DE AGRICULTURA E DO ABASTECIMENTO. Manual genérico de procedimentos para APPCC em indústrias de produtos de origem animal. Disponível em:

$<$ http://pucrs.campus2.br/ thompson/MnualAPPCC.doc>. Acesso em: 5 abril. 2009.

NETO, J. P. C. Segurança e higiene do trabalho em estações de tratamento de água. Dissertação (Mestrado) Universidade Estadual de Campinas, Faculdade de Engenharia Civil, Arquitetura e Urbanismo. Campinas - SP: UNICAMP, 120 p., 2006.

REIS, H. G. dos. Exigências de análise de risco de acidentes, para fins de licenciamento, em instalações que manipulam substancias perigosas, e proposição de abordagem para atendimento. Dissertação (mestrado) Universidade Estadual de Campinas, Faculdade de Engenharia Química. Campinas - SP. UNICAMP, 165 p., 2006.

SENAC/DN. Guia de elaboração do plano APPCC. Rio de Janeiro. SENAC/DN, 314 p., 2001.

SILBERMAN, T. D. de A.; MATTOS, U. A. de O. Metodologia de análise de risco: estudo em uma unidade de cogeração de energia de um shopping center de Macaé. Boletim técnico Organização \& Estratégia, v. 4, n.1, p.155172., 2008.

\section{Dados dos Autores:}

\section{Nome completo: Eduardo Pavan Korf}

Filiação institucional: Universidade de Passo Fundo

Departamento: Faculdade de Engenharia e Arquitetura - FEAR, Curso de Engenharia Ambiental Função ou cargo ocupado: Mestrando PPGEC UFRGS/UPF, bolsista CAPES

Endereço completo para correspondência (bairro, cidade, estado, país e CEP): Universidade de Passo Fundo, Campus I, BR 285, Km 171, Bairro São José, CEP 99052-900 - Passo Fundo - RS

Telefones para contato: Tel.: (54) 3316 8217, Fax: (54) 33168211

e-mail: eduardokorf@gmail.com

\section{Nome completo: Claud Ivan Goellner}

Filiação institucional: Universidade de Passo Fundo

Departamento: Faculdade de Engenharia e Arquitetura - FEAR, Curso de Engenharia Ambiental Função ou cargo ocupado: Professor titular nos cursos de Agronomia e Engenharia Ambiental 
Endereço completo para correspondência (bairro, cidade, estado, país e CEP): Universidade de Passo Fundo, Campus I, BR 285, Km 171, Bairro São José, CEP 99052-900 - Passo Fundo - RS Telefones para contato: Tel.: (54) 3316 8217, Fax: (54) 33168211

e-mail:goellner@upf.br

Enviado em: 26/09/2010

Aprovado em: 27/09/2011 